\title{
CULTURA DE SEGURANÇA DO PACIENTE EM ORGANIZAÇÃO HOSPITALAR*
}

\author{
Ana Cláudia de Azevêdo Bião e Silva ${ }^{1}$, Darci de Oliveira Santa Rosa ${ }^{2}$
}

\begin{abstract}
RESUMO: Estudo do tipo survey realizado em maio de 2015 que descreveu a cultura de segurança do paciente em organização hospitalar. Do total de 511 profissionais de enfermagem, 267 pessoas compuseram a amostra aleatória, 128 atenderam aos critérios de inclusão e responderam ao Questionário "Pesquisa sobre Segurança do Paciente em Hospitais", perfazendo taxa de resposta de 47,94\%. Das dimensões da cultura de segurança, destacaram-se com respostas negativas, "Trabalho em equipe na unidade" com 67\% (338), "Expectativas sobre o seu supervisor/chefe e ações promotoras da segurança do paciente" com 66,3\% (330), "Aprendizado organizacional - melhoria contínua", 53,8\% (198) e "Transferências internas e passagens de plantão" com 50,9\% (255), representando as principais áreas com potencial de melhoria. Não houve dimensões com respostas globais positivas definidas com área de força. Urge responsabilidade compartilhada em implementar estratégias que visem corrigir as fragilidades encontradas em prol de uma assistência qualificada, eficaz, efetiva e segura.
\end{abstract}

DESCRITORES: Cultura; Cultura organizacional; Hospital; Segurança do paciente; Enfermagem.

\section{PATIENT SAFETY CULTURE IN HOSPITAL ORGANIZATION}

ABSTRACT: Survey study conducted in May 2015 that described patient safety culture in hospital organization. Of the total 511 nursing professionals, 267 individuals composed the random sample, 128 met the inclusion criteria and answered the questionnaire "Pesquisa sobre Segurança do Paciente em Hospitais" (Survey on Patient Safety in Hospitals), totaling a response rate of $47.94 \%$. Of the safety culture dimensions, the ones which stood out with negative answers were: "Team work in the unit" with $67 \%$ (338), "Expectations on your supervisor/boss and actions promoting patient safety" with $66.3 \%$ (330), "Organizational learning - continuous improvement" with 53.8\% (198) and "Internal transfers and shift changes" with 50.9\% (255), representing the main areas with potential for improvement. There were no dimensions with positive overall answers defined with area of strength. It is of utmost importance to share responsibility in implementing strategies aiming to correct weaknesses found to promote qualified, effective and safe health care.

DESCRIPTORS: Culture; Organizational culture; Hospital; Patient safety; Nursing.

\section{CULTURA DE SEGURIDAD DEL PACIENTE EN ORGANIZACIÓN HOSPITALARIA}

RESUMEN: Estudio tipo survey realizado en mayo de 2015, describiendo la cultura de seguridad del paciente en organización hospitalaria. Del total de 511 profesionales de enfermería, 267 individuos compusieron la muestra aleatoria; 128 cumplieron los criterios de inclusión y respondieron el Cuestionario "Investigación sobre Seguridad del Paciente en Hospitales", ofreciendo tasa de respuesta del $47,94 \%$. De las dimensiones de la cultura de seguridad, obtuvieron respuestas negativas: "Trabajo en equipo en la unidad", 67\% (330), “Expectativas sobre su supervisor/jefe y acciones promotoras de seguridad del paciente", 66,3\% (330), "Aprendizaje organizacional - capacitación continua", 53,8\% (198) y "Transferencias internas y cambios de guardia", 50,9\% (255); representando las principales áreas con potencial de mejora. No hubo dimensiones con respuestas globales positivas definidas con área de fuerza. Urge responsabilidad compartida para implementar estrategias de corrección de debilidades encontradas, en pro de una atención calificada, eficaz, efectiva y segura.

DESCRIPTORES: Cultura; Cultura Organizacional; Hospitales; Seguridad del Paciente; Enfermería.

\footnotetext{
*Artigo extraído da dissertação intitulada: "Cultura de segurança do paciente em organização hospitalar". Escola de Enfermagem - Universidade Federal da Bahia, 2016.

${ }^{1}$ Enfermeira. Mestranda em Enfermagem pela Escola de Enfermagem da Universidade Federal da Bahia. Secretaria Municipal de Saúde de Salvador. Salvador, BA, Brasil.

Enfermeira. Pós-doutora em Enfermagem. Docente da Escola de Enfermagem da Universidade Federal da Bahia. Salvador, BA, Brasil.
}

Autor Correspondente:

Ana Cláudia de Azevêdo Bião e Silva

Universidade Federal da Bahia

Al. Paradiso, 75 - 41830-620 - Salvador, BA, Brasil

E-mail: anaclaudiabiao@gmail.com
Recebido: 29/02/2016

Finalizado: $22 / 06 / 2016$ 


\section{INTRODUÇÃO}

Nos tempos atuais, a assistência à saúde perpassa não apenas pelos aspectos técnico-científicos, como também por um conjunto de dimensões que explicitam a cultura organizacional de cada serviço de saúde, em particular de cada organização hospitalar. O hospital é considerado, entre as organizações existentes, uma das mais complexas, cujo funcionamento se dá por meio da interação entre os setores envolvidos e a necessidade de harmonizar os processos operacionais para construção do almejado produto final. Em outras palavras, desenvolver ações que promovam, com responsabilidade, qualidade e segurança no atendimento ao paciente que procurou o serviço em busca de bem-estar e saúde. A conjunção simultânea de fatores técnicos, operacionais e ambientais associadas ao potencial humano, tem por objetivo encontrar as melhores práticas para o pronto restabelecimento do paciente e seu mais breve retorno à família e à sociedade ${ }^{(1)}$.

Entende-se cultura de segurança como um dos valores da cultura organizacional cujo objetivo é favorecer ações coerentes e comportamentos mais adequados, tendo atitudes e normas como fundamentais para um ambiente seguro. Mas esta cultura de segurança pode se apresentar diferente entre subgrupos das organizações, como por exemplo, setores e categorias profissionais que possuem sua própria cultura e modus operandi regidos por códigos disciplinares específicos. Além das atitudes e valores individuais dos membros da organização, o comprometimento e envolvimento do staff executivo, tático e operacional com a segurança influenciam diretamente no cotidiano, já que esta precisa ser renovada diariamente devido a sua característica perecível ${ }^{(2)}$.

Define-se segurança do paciente como redução ao mínimo aceitável do risco de dano desnecessário associado ao cuidado de saúde ${ }^{(3)}$. Conforme o The Canadian Patient Safety Dictionary, segurança do paciente corresponde nas organizações de saúde: à redução e mitigação de atos não seguros, de erros não-intencionais; aplicação de boas práticas baseadas nas condições de estrutura e processos de trabalho, dos serviços de saúde, que reduzam os riscos e eventos adversos no cuidado, em prol de obter resultados satisfatórios para os pacientes ${ }^{(4)}$.

Nessa perspectiva, a segurança do paciente torna-se foco de pesquisadores de todo o mundo assumindo uma dimensão fundamental para qualidade em saúde. A Organização Mundial de Saúde (OMS) estabeleceu, em 2004, a Aliança Mundial para a Segurança do Paciente (World Alliance for Patient Safety) ao reconhecer globalmente a magnitude do fenômeno ${ }^{(5)}$.

A cultura de segurança define-se através do comportamento individual e compartilhado dos membros da organização, conforme o comprometimento e responsabilidade destes, na oferta de serviço qualificado e seguro, independente da posição hierárquica ${ }^{(6)}$. Nesta perspectiva

[...] a cultura de segurança do paciente tem recebido crescente atenção no campo das organizações de saúde. Os cuidados de saúde, cada vez mais complexos, elevam o potencial de ocorrência de incidentes, erros ou falhas, particularmente em hospitais. Uma cultura de segurança fortalecida no âmbito hospitalar emerge como um dos requisitos essenciais para melhorar a qualidade do cuidado de saúde (7:152).

Este fato aponta para a necessidade de mudança nas organizações hospitalares, de acordo com cada cultura organizacional, por meio de estratégias de gestão, processos inovadores em prol da cultura de segurança do paciente, que considerem os fatores facilitadores e dificultadores, tais como:

[...] pessoas, ideias, padrões de relacionamento e tempo. Pode-se dizer que em certos ambientes existe uma cultura organizacional que favorece a mudança e a inovação e em outros não. A criação de um ambiente seguro para pacientes e profissionais requer um plano estratégico, liderança e trabalho em equipe ${ }^{(8: 356)}$.

Logo, considera-se uma organização hospitalar segura quando há esforço e cooperação coletiva de todos os profissionais de saúde, em todos os níveis. Para legitimar e fortalecer os esforços internacionais e nacionais em prol da segurança do paciente, o Ministério da Saúde do Brasil instituiu o Programa Nacional de Segurança do Paciente (PNSP) por meio da Portaria $n^{\circ} 529$, de $1^{\circ}$ de abril de 2013.

\section{Este novo programa almeja}

[...] estimular o olhar crítico para a segurança do paciente, com estabelecimento de metas específicas para prevenir danos evitáveis e minimizar riscos de incidentes, propõe-se o desenvolvimento de um programa nacional de segurança do paciente que esteja vinculada aos programas de qualidade do governo federal. Tal programa 
deve envolver, no mínimo, o Ministério da Saúde, a ANVISA, a Agência Nacional de Saúde Suplementar (ANS) e o Ministério da Educação, sendo o último um importante aliado para a formação de profissionais de saúde, especialmente nos hospitais de ensino ${ }^{(9: 793)}$.

O "PNSP visa, especialmente, prevenir, monitorar e reduzir a incidência de EAs nos atendimentos prestados, promovendo melhorias relacionadas à segurança do paciente e a qualidade em serviços de saúde do País" (10:12).

Assim, "conhecer a cultura de segurança de prestadores do cuidado em saúde implica, diretamente, nas atividades de promoção da segurança do paciente" (11:130).

Diante do exposto, definiu-se como objeto de estudo a cultura de segurança do paciente em uma organização hospitalar.

Partiu-se da seguinte questão de pesquisa: "Qual a cultura de segurança do paciente de uma organização hospitalar pública localizada na cidade de Feira de Santana-Bahia?" Foi definido como objetivo descrever a cultura de segurança do paciente de uma organização hospitalar pública localizada na cidade de Feira de Santana-Bahia.

Esta pesquisa contribuirá para: a consolidação do Programa Nacional de Segurança do Paciente (PNSP), ampliação do conhecimento sobre a temática, melhoria dos processos de formação e de capacitação dos profissionais de saúde, melhoria da qualidade da assistência e do gerenciamento hospitalar.

\section{- MÉTODO}

Trata-se de estudo transversal do tipo survey, de natureza descritiva. A organização hospitalar escolhida foi um hospital público geral, de grande porte, referência em média e alta complexidade no Estado da Bahia e cadastrado no SUS (Sistema Único de Saúde) localizado na cidade de Feira de Santana, Bahia, Brasil.

A população foi constituída dos 562 trabalhadores de enfermagem, atuantes na organização hospitalar (154 enfermeiros, 226 técnicos de enfermagem, 177 auxiliares de enfermagem e 05 atendentes de enfermagem).

A amostra não-probabilística, por conveniência, foi composta por enfermeiros, técnicos e auxiliares de enfermagem, convidados aleatoriamente 267 que estavam presentes durante o período de coleta. Destes, 128 constituíram a amostra correspondendo a 47,94\% dos convidados/respondentes. Os participantes foram identificados pela letra "Q" (questionário) acompanhada por numeral arábico, conforme os instrumentos iam sendo devolvidos.

O critério de inclusão dos participantes foi possuir vínculo com a organização hospitalar há pelo menos um ano, por considerar que este período é suficiente para exposição e assimilação da cultura de segurança do paciente nesta organização hospitalar ${ }^{(12-13)}$.

Foram excluídos profissionais que estavam de férias, licença médica, licença maternidade e paternidade, liberados para cursos de longa duração (especialização, mestrado e doutorado). Entre estes um enfermeiro que havia acabado de receber a aposentadoria e outros 50 estavam afastados no mês de maio de 2015 (período da coleta) por motivo de licença médica, prêmio e maternidade e férias. Partiu-se do entendimento de que o distanciamento do profissional da atual dinâmica da organização hospitalar pode interferir nos dados colhidos e nas respostas ao inquérito no momento da pesquisa ${ }^{(14)}$.

A aplicação dos questionários ocorreu na primeira quinzena do mês de maio de 2015, após aprovação da administração do hospital. Para acesso aos participantes foram efetuadas visitas diárias a todas as unidades do hospital, no turno diurno. Os questionários e o TCLE foram entregues pessoalmente pela pesquisadora.

Ao serem devolvidos, os questionários foram numerados e os dados transcritos para o software Microsoft Excel 2010, em tabela adaptada a partir da elaborada pelo Premier, como ferramenta auxiliar das organizações hospitalares especificamente no armazenamento, processamento e análise dos 
dados do questionário "Pesquisa sobre Segurança do Paciente em Hospitais" (HSOPSC).

Foram considerados completos questionários que tiveram suas questões respondidas em $50 \%$ ou mais das opções. A taxa de resposta, isto é, o quantitativo de questionários não respondidos e não encontrados, foi calculada a partir da subtração entre o total de questionários disponibilizados e o total de respondidos. Foram considerados como perdas aqueles questionários preenchidos inadequadamente ou incompletos, ao término de 02 semanas, período do encerramento da coleta de dados.

Após o término da transcrição, os dados foram exportados para o IBM Statistical Package for the Social Sciences 20.0 pelo qual foi realizado o tratamento dos dados.

$\mathrm{Na}$ análise descritiva, as variáveis quantitativas foram representadas por suas médias e desviospadrão quando suas distribuições eram normais e por medianas e quartis quando não apresentavam curva normal. A definição de normalidade foi feita através de análise gráfica e teste de Shapiro-Wilk. As variáveis categóricas foram representadas através de frequências e porcentagens. Variáveis ordinais foram descritas através de tabelas e figuras, quando julgado pertinente, mantendo as categorias originais individualizadas.

As análises por dimensão foram feitas após obtenção dos resultados para as perguntas com ordem inversa, nas quais uma resposta mais baixa indicava maior grau de segurança (sinalizadas com letra "R").

Os resultados da pesquisa estão exibidos da seguinte forma:

- Caracterização das variáveis características profissionais dos participantes: apresentada em forma de tabela com frequência e mediana por categoria profissional dos participantes da pesquisa.

- $\quad$ Respostas às questões por dimensões da cultura de segurança e de resultado: apresentadas graficamente as frequências de respostas positiva/fortemente positiva, neutra ou negativa/fortemente negativa para cada pergunta, no que tange aos resultados globais.

- Composto Scores: frequência de respostas agrupadas por dimensões de cultura de segurança e resultado representada graficamente.

- Foram apresentadas as frequências de respostas positiva/fortemente positiva, neutra ou negativa/fortemente negativa por categoria para cada pergunta, no que tange aos resultados globais dos itens.

No que tange à "Seção A - Sua área/unidade de trabalho", as questões foram correlacionadas às nomenclaturas utilizadas pela organização hospitalar da seguinte forma:

- "a. Diversas unidades do hospital/Nenhuma unidade específica" e "n. Outra, por favor especifique:": Ambulatório, Banco de Leite, Central de Material Esterilizado, Diretoria de Enfermagem, Comissão de Controle de Infecção Hospitalar e Vigilância Epidemiológica - devido ao baixo numero de respondentes para estes;

- “b. Clínica (não cirúrgica): Unidade de Clínica Médica e Clínica Neurológica;

- "c. Cirurgia": Centro Cirúrgico, Clínica Cirúrgica e Clínica Ortopédica;

- “d. Obstetrícia": Centro Obstétrico, Clínica Materno-Infantil e Mãe-Canguru.

- "f. Setor de Emergência": Triagem, Sala Vermelha, Estabilização, Pequena Cirurgia, ProntoAtendimento Feminino, Pronto-Atendimento Masculino, Ortopedia (Emergência).

- $\quad$ g. Unidade de terapia intensiva (qualquer tipo)": UTI 1, UTI 2, UTI Neonatal e Berçário.

- As questões "e. Pediatria", "h. Psiquiatria", “i. Reabilitação", “j. Farmácia”, "k. Laboratório", "I. Radiologia" e "m. Anestesia" não houveram respondentes.

A pesquisa e seu Termo de Consentimento Livre e Esclarecido foram aprovados em 15/04/2015, sob Parecer Consubstanciado $n^{\circ}$ 1.022.831, do Comitê de Ética em Pesquisa da Escola de Enfermagem da Universidade Federal da Bahia. 
Obteve-se uma taxa de resposta de 47,94\% referente aos 128 questionários respondidos. De acordo com a Tabela 1, os participantes do estudo são 97,6\% (121) do sexo feminino, encontramse na faixa etária de 32-44 anos com mediana de 37, e trabalham entre 6 -18 anos, com tempo médio de 10 anos, $58,7 \%$ (74) possui de 1 (um) a 5 (cinco) anos de vínculo com o hospital, 58,3\% (74) possuem de 1 (um) a 5 (cinco) anos de atuação na área/unidade, $70,9 \%$ (90) trabalham de 20 a 39 horas/semana neste hospital.

Do total, 56,5\% (70) são técnicos de enfermagem e $37,1 \%$ (46) são enfermeiras e a maioria informa ter interação ou contato direto com os pacientes, totalizando $94,4 \%$ (118). Sobre o grau de instrução $38,4 \%$ (48) possuem pós-graduação em nível lato sensu e 6,4\% possui pós-graduação stricto sensu.

A Tabela 2 apresenta o local de atuação dos participantes do estudo. A Unidade de Terapia Intensiva se destaca com $24 \%$ (30) profissionais, seguida das unidades de Emergência com 22,4\% (28).

Na Figura 1 verifica-se que 87,8\% (108) dos profissionais informaram não ter feito nenhuma comunicação sobre eventos nos últimos 12 meses.

Na Figura 2 constata-se que a frequência de nota regular para a segurança do paciente, com $47,9 \%$ (56), sendo a maior frequência de respostas nesta questão.

Na Figura 3 observa-se que a composição das respostas agrupadas por dimensões da cultura de segurança, destacam-se, pelo percentual de respostas negativas superioresa $50 \%$, as dimensões "Trabalho em equipe na unidade" com 67\% (338), "Expectativas sobre o seu supervisor/chefe e ações promotoras da segurança do paciente" com 66,3\% (330), "Aprendizado organizacional melhoria contínua", 53,8\% (198) e "Transferências

Tabela 2 - Setor de trabalho da equipe de enfermagem na organização hospitalar. Salvador - BA, Brasil, 2016

\begin{tabular}{lcc} 
Característica & Frequência & Percentual \\
\hline Clínica & 10 & 8 \\
\hline Cirurgia & 22 & 17,6 \\
\hline Obstetrícia & 17 & 13,6 \\
\hline Emergência & 28 & 22,4 \\
\hline UTI & 30 & 24 \\
\hline Outros & 18 & 14,4
\end{tabular}

Tabela 1 - Características sociodemográficas e de vínculo com a organização hospitalar da equipe de enfermagem. Salvador, BA, Brasil, 2016

\begin{tabular}{|c|c|}
\hline Característica & $\begin{array}{c}\text { Total } \\
(N=128)\end{array}$ \\
\hline Sexo feminino & $121(97,6)$ \\
\hline Idade & $37(32-44)$ \\
\hline \multicolumn{2}{|l|}{ Mediana (quartis) } \\
\hline \multicolumn{2}{|l|}{ Cargo } \\
\hline Enfermeiro & $46(37,1)$ \\
\hline Técnico de enfermagem & $70(56,5)$ \\
\hline Auxiliar de enfermagem & $8(6,5)$ \\
\hline \multicolumn{2}{|l|}{ Escolaridade } \\
\hline Segundo grau completo & $36(28,8)$ \\
\hline Ensino superior incompleto & $12(9,6)$ \\
\hline Ensino superior completo & $21(16,8)$ \\
\hline Pós-graduação (Especialização) & $48(38,4)$ \\
\hline $\begin{array}{l}\text { Pós-graduação (Mestrado ou } \\
\text { Doutorado) }\end{array}$ & $8(6,4)$ \\
\hline \multicolumn{2}{|l|}{ Tempo de vinculação } \\
\hline 1 a 5 anos & $74(58,7)$ \\
\hline 6 a 10 anos & $25(19,8)$ \\
\hline 11 a 15 anos & $6(4,8)$ \\
\hline 16 a 20 anos & $8(6,3)$ \\
\hline 21 anos ou mais & $13(10,3)$ \\
\hline \multicolumn{2}{|l|}{ Tempo na área } \\
\hline Menos de 1 ano & $14(11)$ \\
\hline 1 a 5 anos & $74(58,3)$ \\
\hline 6 a 10 anos & $20(15,7)$ \\
\hline 11 a 15 anos & $8(6,3)$ \\
\hline 16 a 20 anos & $5(3,9)$ \\
\hline 21 anos ou mais & $6(4,7)$ \\
\hline \multicolumn{2}{|c|}{ Horas/semana de trabalho neste hospital } \\
\hline Menos de 20 & $3(2,4)$ \\
\hline 20 a 39 & $90(70,9)$ \\
\hline 40 a 59 & $28(22)$ \\
\hline 60 a 79 & $3(2,4)$ \\
\hline 80 a 99 & $1(0,8)$ \\
\hline 100 ou mais & $2(1,6)$ \\
\hline $\begin{array}{l}\text { Interação ou contato direto com } \\
\text { pacientes }\end{array}$ & $118(94,4)$ \\
\hline Tempo de profissão & $10(6-18)$ \\
\hline Mediana (quartis) & \\
\hline
\end{tabular}


internas e passagens de plantão" com 50,9\% (255), representando as principais áreas com potencial de melhoria. Nesta mesma figura, não houve dimensões com respostas globais positivas iguais ou superiores a $75 \%$ definidas com área de força.

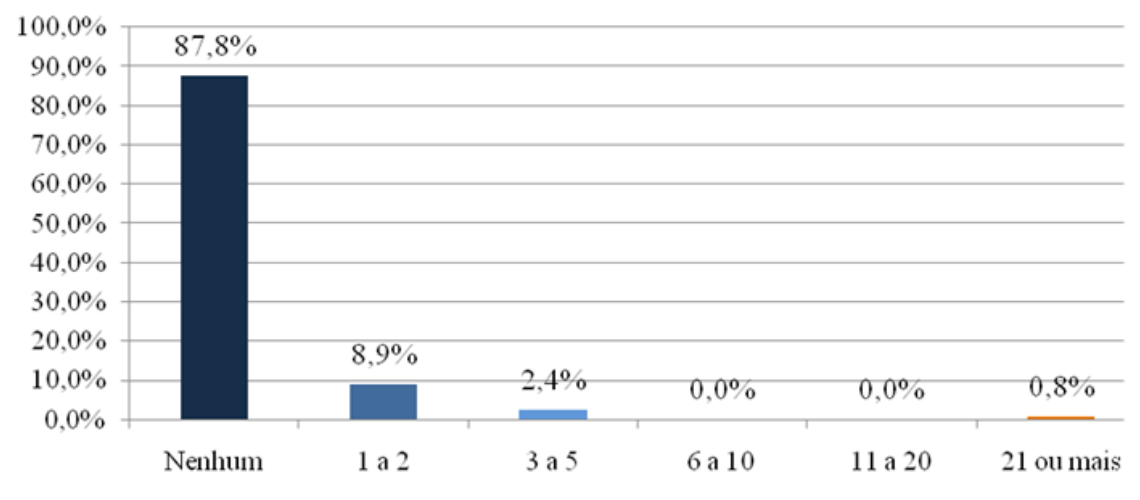

Frequência de eventos relatados

Figura 1 - Frequência de eventos notificados. Salvador - BA, Brasil, 2016

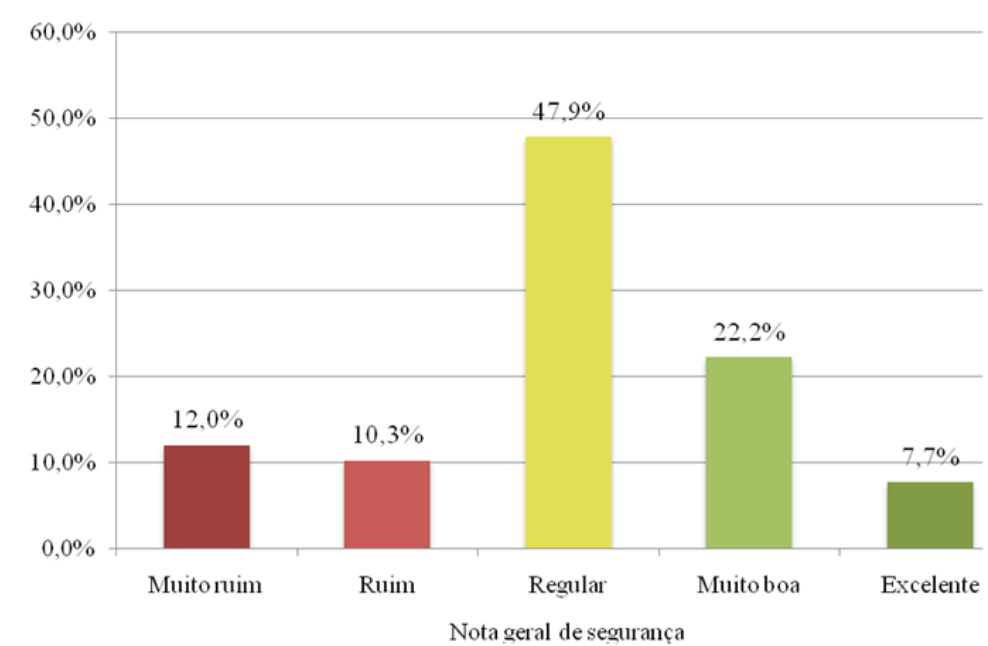

Figura 2 - Nota de segurança do paciente. Salvador, BA, Brasil, 2016

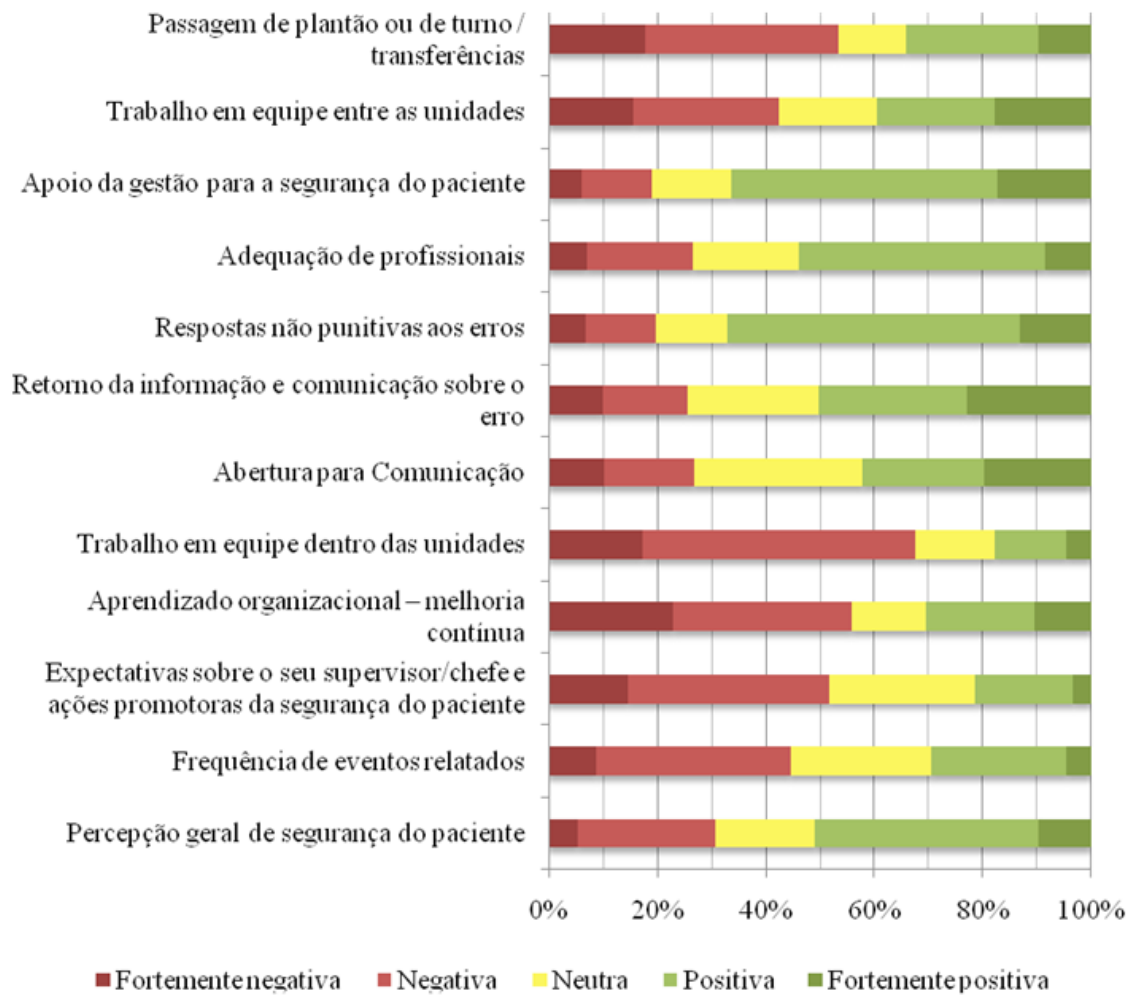

Figura 3 - Composição das respostas agrupadas por dimensões da cultura de segurança de resultado. Salvador, BA, Brasil, 2016 


\section{- DISCUSSÃO}

O perfil dos participantes desta pesquisa difere de estudo realizado em hospital de ensino do estado de São Paulo em 2012, cuja caracterização dos participantes da pesquisa possuía a faixa etária predominante de 26 a 35 anos, denotando um grupo mais jovem de profissionais ${ }^{(15)}$. Nesta pesquisa os profissionais estão na faixa etária superior.

Quanto ao grau de escolaridade, o grupo de enfermeiros desse estudo apresentou resultado significativo quanto à quantidade de profissionais com pós-graduação lato sensu, incluindo aqueles cujo vínculo com a organização hospitalar é como técnico de enfermagem, diferindo do estudo sobre cultura de segurança do paciente em um hospital de ensino cujo número majoritário foi do ensino médio completo ${ }^{(15)}$.

Obteve-se maior quantitativo de técnicos de enfermagem, seguido de enfermeiros e auxiliares de enfermagem com quantitativo pouco expressivo. Já no estudo supracitado ${ }^{(15)}$ a categoria profissional predominante foi de auxiliares de enfermagem, seguida de técnicos de enfermagem, e por fim a de enfermeiros ${ }^{(15)}$.

Quanto ao sexo dos profissionais, o maior percentual foi do sexo feminino, característico da profissão e corrobora com a pesquisa já citada ${ }^{(15)}$ que apresenta também a maioria foi do sexo feminino (74,5\%), contudo já se observa inserção mais significativa do sexo masculino.

Quanto à distribuição dos participantes nas unidades do hospital, este estudo se diferencia do estudo sobre a cultura de segurança do paciente em hospitais espanhóis em que a unidade mais participante foi a Clínica (não-cirúrgica) ${ }^{(16)}$.

A maioria dos participantes desta pesquisa tinham contato direto com os pacientes, assemelhandose aos achados do referido estudo espanhol com $92,2 \%{ }^{(16)}$. Mas parte dos participantes trabalham de 20 a 39 horas/semana no hospital, de acordo com os regimes contratuais de carga horária da enfermagem com contratos de 20 horas, 30 horas e 36 horas semanais, ratificando os achados do estudo do Ministério de la Sanidad y Consumo ${ }^{(16)}$ cujos resultados estão acima desta faixa com 57,2\%.

Nesta pesquisa a maioria dos profissionais possuem de 1 (um) a 5 (cinco) anos de vínculo com o hospital, diverge do estudo do Ministerio de la Sanidad y Consumo ${ }^{(16)}$, em que 23,2\% possui 21 anos ou mais de vínculo com o hospital.

Quanto ao tempo na área/unidade, um maior percentual possui de 1 (um) a 5 (cinco) anos diferindo do estudo do Ministério de la Sanidad y Consumo ${ }^{(16)}$, em que 20,0\% atuavam entre 6 a 10 anos na mesma unidade.

Sobre notificações de eventos nos últimos 12 meses, encontrou-se vulnerabilidade significativa, pois a não-informação foi elevada $(87,8 \%)$. Logo, infere-se que uma cultura organizacional punitiva, pautada na culpabilização, "poderá causar omissão dos relatos desses eventos, dificultan $\neg$ do a construção de uma cultura institucional voltada para a segurança do paciente" (17:278). No estudo da Fiocruz, obtevese resultado semelhante: "78\% relataram nenhum evento nos últimos 12 meses, configurando forte assimetria na reposta a esse item"(7:80).

A percepção geral de segurança apresentou-se pela frequência de nota regular (47,9\%), sendo a maior frequência de respostas nesta questão. Este valor, muito próximo a $50 \%$, se somado aos $10,3 \%$ que consideraram ruim e $12 \%$ muito ruim, considera-se, de modo geral, frágil a segurança do paciente na organização pesquisada. Portanto, corrobora-se com estudos que salientam a necessidade de implantar estratégias voltadas para melhoria da qualidade assistencial, associadas a mecanismos de controle e monitorização das ações, juntamente com compromisso, transparência e responsabilidade coletiva para subsidiar a gestão de riscos e alcançar melhor percepção da segurança do paciente ${ }^{(8)}$.

A dimensão "Trabalho em equipe na unidade" apresentou resultados globais de respostas negativas expressivos, logo apresenta-se com área com potencial de melhoria. O estudo de revisão sobre o aspecto atual da cultura de segurança do paciente ${ }^{(18)}$ destaca que o trabalho em equipe associado ao comprometimento individual de cada profissional confirma o princípio de Hipócrates de não causar dano ao paciente, considerado um fator de proteção para segurança do paciente. 
O trabalho em equipe integrativo e cooperativo entre profissionais de enfermagem, assim como o respeito mútuo aos limites e tempo individual de cada um na realização de tarefas, são essenciais para subsidiar o cuidado seguro e qualificado pela Enfermagem, bem como alto grau de satisfação dos profissionais e redução das taxas de mortalidade ${ }^{(13)}$.

No que tange à dimensão "Expectativas sobre o seu supervisor/chefe e ações promotoras da segurança do paciente", o percentual significativo de respostas negativas leva a classificar esta dimensão como área com potencial de melhoria. Estudo bibliométrico sobre cultura de Segurança do paciente em instituições de saúde salienta que cabe aos líderes oferecer suporte necessário para que sua equipe possa "[...] trabalhar em condições adequadas, que minimizem a ocorrência de erros e de danos para os usuários do serviço"(8).

Nessa perspectiva, enfermeiros, enquanto líderes da equipe de enfermagem, devem capitanear, incentivar e apoiar os membros de sua equipe no desenvolvimento dos processos assistenciais em prol da segurança, favorecendo a obtenção dos melhores resultados para o paciente. No interior das organizações hospitalares, os enfermeiros representam o principal elo no desempenho das ações, sendo responsáveis pela garantia dos recursos necessários, identificar e promover valores coletivos, defender e moldar a cultura organizacional para o seu bom desempenho ${ }^{(13)}$.

A dimensão "Aprendizado organizacional - melhoria contínua" cujo quantitativo de respostas negativas foi consideravelmente representativo, mesmo com percentuais individuais das respostas sem destaque, conforme critérios preconizados, foi considerada área com potencial de melhoria.

Esta dimensão exibe o envolvimento organizacional frente à busca contínua por soluções para sanar as dificuldades que comprometam a melhoria da assistência. Este envolvimento evolui a partir do momento que a cultura de segurança do paciente amadurece. Tal amadurecimento é explicitado pelas ações proativas de identificação e instituição de ações que minimizem os riscos e eventos, entendendo-as com oportunidades de melhoria, numa perspectiva educativa constante ${ }^{(19)}$.

As características das organizações hospitalares estão relacionadas às suas respectivas culturas. Isto impacta na qualidade da assistência e nas tomadas de decisão estratégicas pelos gestores hospitalares que devem ser alicerçadas na compreensão dos fatores, processos e direcionadas a manter serviços de saúde seguros para seus pacientes. Para tal, estes gestores devem implementar ações que visem prevenir, detectar e atenuar riscos de danos a que os pacientes estão expostos ${ }^{(8)}$.

Nesta perspectiva, profissionais e gestores hospitalares devem estar comprometidos em garantir uma assistência segura para os pacientes e os profissionais de saúde, com a promoção do aprendizado organizacional, da melhoria contínua das práticas assistenciais e gerenciais nas organizações hospitalares ${ }^{(17)}$.

A dimensão "Transferências internas e passagens de plantão" obteve respostas negativas também vultosas, indicativas de área com potencial de melhoria. O estudo sobre a comunicação na passagem de plantão da equipe de enfermagem e os fatores relacionados à segurança do paciente ${ }^{(20)}$ afirma que "os comportamentos/atitudes dos profissionais durante as passagens de plantão e os conhecimentos/ percepções dos profissionais quanto à importância da comunicação durante as passagens de plantão e à segurança do paciente" interferem na segurança do paciente. Constata-se que a segurança do paciente pode estar comprometida nesta organização hospitalar.

\section{CONSIDERAÇÕES FINAIS}

De acordo com os resultados descritivos, não foram identificadas áreas de força. Foram identificadas 04 áreas com potencial de melhoria.

Uma organização hospitalar cuja cultura de segurança do paciente ao ser descrita não apresenta nenhuma dimensão como área de força (75\% ou mais de respostas positivas) e apresenta 04 de 12 das suas dimensões como áreas prioritárias com potencial de melhoria (50\% ou mais de respostas negativas), entende-se que esta organização possui uma cultura de segurança fragilizada, de caráter punitivo face à falibilidade humana. Este fato inibe a notificação dos erros, a oportunidade de aprimoramento da equipe, e a promoção de uma assistência à saúde segura. 
Constata-se que a segurança do paciente está comprometida nesta organização hospitalar. Isso requer maior envolvimento dos profissionais desta organização no desenvolvimento de estratégias para o aprendizado organizacional e melhoria contínua, alicerçadas no Programa Nacional de Segurança do Paciente.

Sugere-se minimizar fragilidades, melhorar a qualidade e segurança dos serviços ofertados, compartilhar responsabilidades entre todos os envolvidos no cuidado, direta ou indiretamente, para garantir a segurança do paciente nesta organização.

Esta pesquisa teve como limitações: a restrição de acesso à população no período noturno e finais de semana, dificuldade de maior adesão da população devido à extensão do questionário, assim como algumas questões de difícil compreensão.

\section{REFERÊNCIAS}

1. Marx LC. Manual de gerenciamento de enfermagem. $2^{a}$ ed. São Paulo: EPUB; 2003.

2. Vincent C. Segurança do paciente: orientações para evitar eventos adversos. Trad. Rogério Videira. São Caetano do Sul, SP: Yendis Editora; 2009.

3. Ministério da Saúde (BR). Portaria n. 529 de 1 de abril de 2013. Institui o Programa Nacional de Segurança do Paciente. Diário Oficial da União da República Federativa do Brasil, Brasília, 1 de abr. de 2013.

4. Davies JM, Hébert P, Hoffman C. The Canadian Patient Safety Dictionary. Ottawa, Canada: Royal College of Physicians and Surgeons of Canada; 2003. [acesso 10 ago 2015] Disponível: http://www.royalcollege.ca/portal/ page/portal/rc/common/documents/publications/patient_safety_dictionary_e.pdf

5. Reis CT, Martins M, Laguardia J. A segurança do paciente como dimensão da qualidade do cuidado de saúde: um olhar sobre a literatura. Ciênc. saúde coletiva. [Internet] 2013; 18(7) [acesso em 17 nov 2013]. Disponível: http://dx.doi.org/10.1590/S1413-81232013000700018

6. Agência Nacional de Vigilância Sanitária (ANVISA) [Internet]. Assistência segura: uma reflexão teórica aplicada à prática; 2013. [acesso 22 set 2013]. Disponível: http://www.anvisa.gov.br/hotsite/segurancadopaciente/ documentos/junho/Modulo\%201\%20-\%20Assistencia\%20Segura.pdf

7. Reis CT. A cultura de segurança do paciente: validação de um instrumento de mensuração para o contexto hospitalar brasileiro [tese]. Rio de Janeiro (RJ): Escola Nacional de Saúde Pública Sergio Arouca; 2013. Disponível: http://proqualis.net/sites/proqualis.net/files/000002441PwFnHv.pdf

8. Tobias, QGC, Bezerra ALQ, Branquinho NCS, Silva AEBC. Cultura de Segurança do paciente em instituições de saúde: um estudo bibliométrico. Enferm glob. [Internet]. 2014; 13(33) [acesso 22 fev 2014]. Disponível:

http://scielo.isciii.es/pdf/eg/v13n33/pt_revision1.pdf

9. Capucho HC, Cassiani SHB. Necessidade de implantar programa nacional de segurança do paciente no Brasil. Rev Saúde Pública. [Internet] 2013; 47(4) [acesso 11 ago 2015]. Disponível:

http://dx.doi.org/10.1590/S0034-8910.2013047004402

10. Agência Nacional de Vigilância Sanitária (ANVISA) [Internet]. Implantação do Núcleo de Segurança do Paciente em Serviços de Saúde - Série Segurança do Paciente e Qualidade em Serviços de Saúde; 2014. [acesso em 11 mar 2014]. Disponível: http://portalpbh.pbh.gov.br/pbh/ecp/files.do?evento=download\&urlArqPIc=modu lo-6-implantacao-nucleo-de-seguranca-do-paciente.pdf

11. Cauduro FLF, Sarquis LM, Sarquis LMM, Cruz EDA. Cultura de segurança entre profissionais de centro cirúrgico. Cogitare enferm. [Internet] 2015;20(1) [acesso 22 fev 2016]. Disponível: http://dx.doi.org/10.5380/ce.v20i1.36645

12. Sexton JB, Helmereich RL, Neilands TB, Rowan K, Vella K, Boyden J, et al. The safety attitudes questionnaire: psychometric properties, benchmarking data, and emerging research. BMC Health Serv Res. [Internet] 2006; 44(6) [acesso em 05 jul 2015]. Disponível: https://dx.doi.org/10.1186/1472-6963-6-44

13. Oliveira RM, Leitão IMTA, Aguiar LL, Oliveira ACS, Gazos DM, Silva LMS, Barros AA, Sampaio RL. Evaluating 
the intervening factors in patient safety: focusing on hospital nursing staff. Rev. esc. enferm. USP. [Internet] 2015; 49(1) [acesso 27 jun 20125]. Disponível: http://dx.doi.org/10.1590/S0080-623420150000100014

14. Schwonke CRGB. Conhecimento da equipe de enfermagem e cultura de segurança: análise sistêmica dos riscos na assistência ao doente crítico em ventilação mecânica invasiva [tese]. Rio Grande (RS): Universidade Federal do Rio Grande, 2012. Disponível: http://educacao.fau.com.br/site/arquivos/arquivo_20130625152700.pdf

15. da Silva-Batalha EMSS, Melleiro MM. Cultura de segurança do paciente em um hospital de ensino: diferenças de percepção existentes nos diferentes cenários dessa instituição. Texto contexto - enferm. [Internet] 2015; 24(2) [acesso 10 jul 2015]. Disponível: http://dx.doi.org/10.1590/0104-07072015000192014

16. Ministerio de Sanidad y Consumo (ES) [Internet]. Análisis de la cultura sobre seguridad del paciente en el ámbito hospitalario del Sistema Nacional de Salud español; 2008. [acesso 21 fev 2016]. Disponível: http://www.um.es/calidadsalud/archivos/Analisis_cultura_SP_ambito_hospitalario.pdf

17. Françolin L, Gabriel CS, Bernardes A, Silva AEBC, Brito MFP, Machado JP. Gerenciamento da segurança do paciente sob a ótica dos enfermeiros. Rev Esc Enferm USP. [Internet] 2015; 49(2) [acesso 27 jun 2015]. Disponível: https://dx.doi.org/10.1590/S0080-623420150000200013

18. Ramírez OJG, Gámez AS, Gutiérrez AA, Salamanca JG, Veja AG, Galeano EM. Una mirada actual de la cultura de seguridad del paciente. Avances en enfermería. [Internet] 2011; 29(2) [acesso 27 jun 2015]. Disponível: http://www.scielo.org.co/pdf/aven/v29n2/v29n2a15.pdf

19. Peralta TJA. Segurança do Doente - Perspetiva dos Profissionais de um Hospital da Região Centro [dissertação]. Cimbra (PT): Escola Superior de Enfermagem de Coimbra; 2012. Disponível:

http://repositorio.esenfc.pt/private/index.php?process=download\&id=24072\&code $=32$

20. Gonçalves MI. Comunicação na passagem de plantão da equipe de enfermagem em unidades de cuidados intensivos neonatais e os fatores relacionados à segurança do paciente. [dissertação]. Florianópolis (SC): Universidade Federal de Santa Catarina, 2012. Disponível:

https://repositorio.ufsc.br/xmlui/bitstream/handle/123456789/106764/318655.pdf?sequence=1\&isAllowed=y 